\title{
Two troponins, one troponin, none... the dawn of troponin-less decision aids
}

\author{
Richard Body ${ }^{1,2,3} \cdot$ Kerstin De Wit ${ }^{4}$
}

Received: 2 December 2021 / Accepted: 7 December 2021

(C) The Author(s), under exclusive licence to Canadian Association of Emergency Physicians (CAEP)/ Association Canadienne de Médecine d'Urgence (ACMU) 2021

Keywords Acute coronary syndromes $\cdot$ Biomarkers $\cdot$ Sensitivity and specificity $\cdot$ Troponin

Our approach to the diagnosis of acute coronary syndromes (ACS) in the Emergency Department (ED) has undergone a genuine revolution over the past 2 decades. Gone are the days when suspicion of ACS would routinely lead to patients undergoing serial troponin testing over prolonged periods, often with routine telemetry and inpatient imaging thereafter. The advent of high-sensitivity cardiac troponin (hs-cTn) assays enabled us to safely reduce the time interval between serial samples to as little as $1 \mathrm{~h}$. Many patients can now have ACS ruled out following a single blood test, either using very hs-cTn low cut-offs or using validated decision aids such as the HEART score [1], Troponin-only Manchester Acute Coronary Syndromes (T-MACS) decision aid [2], or the Emergency Department Assessment of Chest Pain Score (EDACS) [3].

In this issue, O'Rielly et al. have reported an external validation of a modified HEART score, named the HEAR score, in which a HEAR score of $\leq 1$ could allow ACS to be ruled out without any troponin testing [4]. By scoring elements of a patient's history, ECG, age, and risk factors for cardiovascular disease, the external validation study presented in this issue suggests that the HEAR score could

Richard Body

Richard.body@manchester.ac.uk

1 Emergency Department, Manchester Royal Infirmary, Central Manchester University Hospitals NHS Foundation Trust, Manchester Academic Health Science Centre, Oxford Road, Manchester M13 9WL, UK

2 Division of Cardiovascular Sciences, Core Technology Facility, Manchester, UK

3 Healthcare Sciences Department, Manchester Metropolitan University, Manchester, UK

4 Department of Emergency Medicine, Queen's University, Kingston, ON, Canada instantly 'rule out' ACS in $18 \%$ of patients, achieving a sensitivity of $99 \%$ (95\% CI 96-100\%). This is an important advance for emergency physicians, since it allows the physician to safely withhold troponin testing in patients who are unlikely to have an acute coronary syndrome. Because many of these patients are young and otherwise healthy, they may not require blood testing at all. In turn, this reduces the time spent in the ED and helps address ED crowding.

This study is now one of several validating the HEAR score, which appear to consistently show high diagnostic sensitivity [5]. Like the HEART score, the Troponin-only Manchester Acute Coronary Syndromes (T-MACS) decision aid can be used to 'rule out' ACS following a single measurement of hs-cTn in the ED [3]. Calculators such as MDCalc (https://www.mdcalc.com/troponin-manchesteracute-coronary-syndromes-t-macs-decision-aid) are a great way to calculate the T-MACS score. T-MACS has also recently been modified to produce a version that does not require troponin testing [6]. A recent validation study suggests that the History and ECG Manchester Acute Coronary Syndromes (HE-MACS) decision aid could rule out $13.5 \%$ of patients with a reported sensitivity of $100 \%$ (95\% CI 92-100\%) [7].

While ED visits for chest pain increase each year, the proportion of chest pain patients diagnosed with illness decreases [8]. A rapid rule-out strategy for ACS is more helpful now than ever before. Perhaps, the greatest impact could be seen by deploying these decision aids at the front door of the ED, for example as part of a 'rapid assessment' model (sometimes referred to as 'Doc in the box') whereby clinicians provide an initial assessment upon a patient's arrival. If the history and ECG could be used to identify a proportion of patients who do not require blood testing to 'rule out' ACS, then some patients could be discharged quickly, unburdening crowded EDs. Other important uses 
could relate to environments where cardiac troponin testing is not readily available, for example urgent or urgent care centres or for patients receiving an emergency ambulance response in the pre-hospital environment. In such contexts, troponin-less decision aids such as HEAR or HE-MACS could rapidly obviate the need for patients to be transported to hospital, or triage the urgency of transport, freeing up emergency medical services for those who most need them.

With a wealth of data from observational studies in the EDs, it is now time to study the real-world impact of implementing ED decision aids. Should implementation be successful, we will have witnessed another important step change in our approach to the diagnosis of ACS: the introduction of dynamic evidence-based risk stratification, where validated decision aids are used to guide clinical decisionmaking based on the available information at any given time point. At each stage of the patient journey and even with what we would ordinarily consider to be incomplete information, decision aids will identify some patients who can safely exit the testing pathway, while determining the next required investigation for those who have not had the diagnosis 'ruled out'. The dawn of an exciting new era of dynamic risk stratification is here.

Acknowledgements Richard Body's institution has received research grants from Abbott Point of Care and Roche Diagnostics and has received donation of reagents for research from Alere, FABPulous BV, and Siemens Healthineers. Richard Body has undertaken consultancy with Roche, Siemens, Abbott, Beckman Coulter, LumiraDx, Aptamer Group, Creavo, and Radiometer. Kerstin de Wit has received a research grant from Bayer.

\section{References}

1. Van Den Berg P, Body R. The HEART score for early rule out of acute coronary syndromes in the emergency department: a systematic review and meta-analysis. Eur Heart J Acute Cardiovasc Care. 2018;7:111-9.

2. Body R, Carlton E, Sperrin M, Lewis PS, Burrows G, Carley S, McDowell G, Buchan I, Greaves K, Mackway-Jones K. Troponinonly Manchester Acute Coronary Syndromes (T-MACS) decision aid: single biomarker re-derivation and external validation in three cohorts. Emerg Med J. 2017;34:349.

3. Boyle RSJ, Body R. The Diagnostic Accuracy of the Emergency Department Assessment of Chest Pain (EDACS) Score: a systematic review and meta-analysis. Ann Emerg Med. 2021;77:433-41.

4. O'Rielly S, Andruchow JE, McRae AD. External validation of a low HEAR score to identify emergency department chest pain patients at very low risk of major adverse cardiac events without troponin testing. Can J Emerg Med (in press)

5. Smith LM, Ashburn NP, Snavely AC, Stopyra JP, Lenoir $\mathrm{KM}$, Wells BJ, et al. Identification of very low-risk acute chest pain patients without troponin testing. Emerg Med J. 2020;37(11):690-5.

6. Alghamdi A, Howard L, Reynard C, Moss P, Jarman H, MackwayJones K, et al. Enhanced triage for patients with suspected cardiac chest pain: the History and Electrocardiogram-only Manchester Acute Coronary Syndromes decision aid. Eur J Emerg Med Off J Eur Soc Emerg Med. 2019;26(5):356-61.

7. Todd F, Duff J, Carlton E. Identifying low-risk chest pain in the emergency department without troponin testing: a validation study of the HE-MACS and HEAR risk scores. Emerg Med J. 2021. https://doi.org/10.1136/emermed-2021-211669. Accessed 09 Nov 2021.

8. Aalam AA, Alsabban A, Pines JM. National trends in chest pain visits in US emergency departments (2006-2016). Emerg Med J. 2020;37:696. 